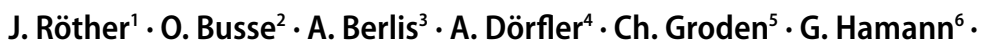

0 . Jansen ${ }^{7} \cdot$ J. Meixensberger ${ }^{8} \cdot$ O. Müller ${ }^{9} \cdot J$. Regelsberger $^{10} \cdot$ H. Steinmetz ${ }^{11} \cdot$

H. Vatter ${ }^{12} \cdot$ W. Weber ${ }^{13} \cdot$ D. Hänggi ${ }^{14} \cdot$ D. Nabavi ${ }^{15}$

'Kopf- und Neurozentrum, Neurologische Abteilung mit überregionaler Stroke Unit, Neurophysiologie und Neurologischer Intensivmedizin, Asklepios Klinik Hamburg Altona, Asklepios Campus Hamburg der Semmelweis Universität, Hamburg, Deutschland; ${ }^{2}$ Deutsche Schlaganfall-Gesellschaft, Berlin, Deutschland; ${ }^{3}$ Diagnostische und Interventionelle Radiologie und Neuroradiologie, Universitätsklink Augsburg, Augsburg, Deutschland; ${ }^{4}$ Neuroradiologische Abteilung, Universitätsklinikum Erlangen, Friedrich-Alexander-Universität Erlangen-Nürnberg, Erlangen, Deutschland; ${ }^{5}$ Universitätsklinikum Mannheim, Medizinische Fakultät Mannheim, Universität Heidelberg, Mannheim, Deutschland; ${ }^{6}$ Klinik für Neurologie und Neurologische Rehabilitation, Bezirkskrankenhaus Günzburg der Bezirkskliniken Schwaben, Günzburg, Deutschland; ${ }^{7}$ Klinik für Radiologie und Neuroradiologie, Universitätsklinikum Schleswig-Holstein Campus Kiel, Kiel, Deutschland; ${ }^{8} \mathrm{Klinik}$ und Poliklinik für Neurochirurgie, Universitätsklinikum Leipzig AöR, Leipzig, Deutschland; ${ }^{9} \mathrm{Klinik}$ für Neurochirurgie, Klinikum Dortmund, Dortmund, Deutschland; ${ }^{10}$ Neurochirurgische Klinik, Universitätsklinikum Hamburg Eppendorf, Hamburg, Deutschland; "Klinik für Neurologie, Universitätsklinikum, Goethe-Universität Frankfurt, Frankfurt am Main, Deutschland; ${ }^{12}$ Klinik und Poliklinik für Neurochirurgie, Universitätsklinikum Bonn, Bonn, Deutschland; ${ }^{13}$ Institut für Diagnostische und Interventionelle Radiologie, Neuroradiologie und Nuklearmedizin, Universitätsklinikum Knappschaftskrankenhaus Bochum GmbH, Bochum, Deutschland; ${ }^{14}$ Neurochirurgische Klinik / Department of Neurosurgery, Universitätsklinikum Düsseldorf / Düsseldorf University Hospital, Heinrich-Heine-Universität, Düsseldorf, Deutschland; ${ }^{15}$ Klinik für Neurologie, Vivantes Klinikum Neukölln, Berlin, Deutschland

\title{
Interdisziplinäre neurovaskuläre Netzwerke: eine Standortbestimmung
}

\section{Hintergrund}

Nach einer Pilotphase in den Jahren 2012 bis 2017, an der 10 Universitätskliniken und 6 Krankenhäuser der Maximalversorgung teilnahmen, wurden von 2018 bis 2020 bisher 15 neurovaskuläre Netzwerke (NVN) auditiert und zertifiziert.

Die Bildung interdisziplinärer NVN ergab sich aus der rasanten Weiterentwicklung der Neuromedizin auf den Gebieten der endovaskulären Therapie (mechanische Thrombektomie, interventionelle Aneurysmatherapie mittels Coils, Stents, Flow-Divertern und WebDevices) und der neurochirurgischen Behandlung komplexer Gefäßerkrankungen, die eine Differenzierung der Versorgungsstrukturen mit einer dritten Ebene (regionale Stroke-Unit, überregionale Stroke-Unit und NVN) sinnvoll erscheinen ließ.

Fachvertreter der drei Neuro-Fächer (Neurologie, Neurochirurgie, Neuroradiologie) und kooperierender Fachgebie- te (Kardiologie, Gefäßchirurgie) verfassten daher ein Strukturpapier, das einerseits das neurovaskuläre Netzwerk definierte und andererseits eine Grundlage für die Zertifizierung von Netzwerkstrukturen schuf [1].

Laut dieser Definition ist ein interdisziplinäres neurovaskuläres Netzwerk eine überregionale Versorgungsstruktur, in der alle Patienten mit neurovaskulären Erkrankungen, besonders auch seltenen Entitäten, interdisziplinär auf hohem Niveau unter besonderer Berücksichtigung innovativer Therapiekonzepte versorgt werden. In diesem NVN leitet ein koordinierendes neurovaskuläres Zentrum mit eigenständigen Abteilungen für Neurologie (mit überregionaler StrokeUnit), Neurochirurgie, Neuroradiologie, Kardiologe und Gefäßchirurgie einen Netzwerkverbund mit Partnerkliniken, um eine optimale Versorgung schwer betroffener Schlaganfallpatienten, Patienten mit Subarachnoidalblutung, Hirnblutungen, Gefäßmissbildungen und
Stenosen der zuführenden Hirnarterien zu gewährleisten (•Infobox 1).

Während die Versorgung neurochirurgischer und gefäßchirurgischer Patienten lokal oftmals auf eine lange Tradition der Zuweisung und Kooperation zurückblicken kann, existierte auf der Ebene der regionalen und überregionalen Stroke-Units vielerorts keine geordnete Netzwerkstruktur für komplexe Gefäßpatienten. Die rasante Entwicklung der mechanischen Thrombektomie nach Publikation der positiven randomisierten, kontrollierten Studien ab 2015 erforderte ein flächendeckendes Konzept mit verbindlichen, strukturierten Zuweisungsregeln, um die Thrombektomie möglichst allen infrage kommenden Schlaganfallpatienten zeitnah und geordnet zukommen zu lassen $[2,3]$.

Vorbilder für die Etablierung von NVN waren Tumor- und Traumazentren, die an größeren Kliniken und insbesondere in Universitätskliniken eine Plattform zur spezialisierten Behandlung 


\section{Infobox 1. Mindestanforde- rungen an ein koordinierendes Zentrum im neurovaskulä- ren Netzwerk. (Auswahl aus dem aktuellen Antrag unter https://www.dsg-info.de/stroke- units/zertifizierungsantraege- zertifizierungskriterien.html) \\ - Eigenständige Abteilungen für Neuro- logie, Neurochirurgie, Neuroradiologie, Kardiologe und Gefäßchirurgie \\ - Mindestens drei Partnerkliniken mit einer zertifizierten Stroke-Unit \\ - Administrative Voraussetzungen (Ge- schäftsordnung, Kooperationsverträge, gemeinsame Manuale/Standards/Treffen/ Konferenzen) \\ - Versorgung von mehr als 1000 Schlagan- fallpatienten oder mehr als 12 Stroke-U- nit-Betten \\ - Neurointensivmedizin mit >200 neurolo- gischen Intensivpatienten/Jahr \\ - Weiterbildungsbefugnis Intensivmedizin \\ - Mindestens drei durch die Deutsche Ge- sellschaft für Interventionelle Radiologie (DeGIR) zertifizierte Neurointerventiona- listen \\ - Mindestens 75 akute endovaskuläre Rekanalisationen und 50 endovaskuläre Aneurysmabehandlungen}

komplexer Patienten bieten. Vorreiter eines gut funktionierenden Netzwerkes war dabei das „Neurovaskuläre Netz Ruhr“, das in einer großen, bevölkerungsreichen Region wie dem Ruhrgebiet eine abgestimmte Versorgung akuter Schlaganfallpatienten etabliert hat [4]. Ziel des Neurovaskulären Netz Ruhr war es, eine verlässliche Kooperationsgrundlage $\mathrm{zu}$ schaffen, gemeinsame Qualitäts- und Therapiestandards zu gewährleisten, die technischen Voraussetzungen für einen teleradiologischen Datentransfer zu etablieren und mittels einer Datenbank eine Qualitätssicherung zu ermöglichen.

Wir berichten nachfolgend über den aktuellen Stand der NVN in Deutschland und liefern eine Leistungsanalyse der 15 bisher zertifizierten NVN.

\section{Daten und Methoden}

Die Daten der Auditberichte der 15 zertifizierten neurovaskulären Netzwerke (NVN) wurden in eine Excel-Tabelle übertragen und ausgewertet (Mittelwert, Median, Spannweite). Weiterentwick- lungen der NVN nach Empfehlungen und Forderungen der Auditoren, die nach der Beendigung des Audits und der Zusendung des Auditberichtes erfolgten, konnten nicht berücksichtigt werden. Die Datenlage entspricht dem Stand des Jahres vor dem Audit, d.h. dass die Daten überwiegend auf die Jahre 2017 bis 2019 zurückgehen. Es handelt sich um den Selbstreport von Daten des Krankenhauscontrollings.

Das Audit wurde jeweils von einem Vertreter der drei Neuro-Fächer und einem leitenden Auditor der LGA InterCert durchgeführt. Der Auditbericht wurde im Mehraugenprinzip von Vertretern des Zertifizierungsausschusses geprüft (die Autoren entsprechen den Mitgliedern des Zertifizierungsausschusses und sind die Fachvertreter ihrer Fachgesellschaften). Während des Audits erfolgten Plausibilitätskontrollen der Zahlenangaben und eine detaillierte Prüfung der Netzwerkstrukturen.

\section{Ergebnisse}

Vier koordinierende Zentren der NVN sind nichtuniversitäre Kliniken der Maximalversorgung, die übrigen 11 sind Universitätskliniken. Die Auditierung der 15 neurovaskulären Netzwerke (NVN) fand zwischen Januar 2018 und Dezember 2019 statt. Die Mehrzahl der NVN besteht aus einem koordinierenden Zentrum und einer unterschiedlichen Anzahl von Partnerkliniken mit regionalen oder überregionalen StrokeUnits, neurologischen Abteilungen ohne zertifizierte Stroke-Unit und internistischen Kliniken. Bei 4 NVN kooperieren auf der Ebene der koordinierenden Zentren 2 bzw. 3 Kliniken, die alle oder fast alle Kriterien eines koordinierenden Zentrums erfüllen und aufgrund der größeren geographischen Reichweite im Einzelfall sehr viele Partnerkliniken umfassen (bis zu 16 Kliniken).

Die 15 NVN behandelten insgesamt 86.510 Schlaganfallpatienten (ischämische Schlaganfälle, intrazerebrale Blutungen und Subarachnoidalblutungen). Die 107 Partnerkliniken lagen im Mittelwert $25 \mathrm{~km}$ (Spanne 4-147km) vom koordinierenden Zentrum entfernt und verlegten 2726 Patienten. Weitere Details zu den verlegten Patienten sind in der - Tab. 2 aufgeführt.

\section{Charakterisierung der koordinierenden Zentren der neurovaskulären Netzwerke}

Die koordinierenden Zentren der neurovaskulären Netzwerke behandelten 18.206 ischämische Schlaganfallpatienten (• Tab. 1). Sie verfügten durchschnittlich über 13 Stroke-Unit-Betten (8-18), 8 Zentren betrieben eine eigenständige neurologische und/oder neurochirurgische Intensivstation. Die neurologischen Abteilungen führten jährlich im Mittel 170 (40-384) systemische Thrombolysen durch. Es erfolgten insgesamt 
J. Röther · O. Busse · A. Berlis · A. Dörfler · C. Groden · G. Hamann · O. Jansen · J. Meixensberger · O. Müller · J. Regelsberger · H. Steinmetz · H. Vatter $\cdot$ W. Weber $\cdot$ D. Hänggi · D. Nabavi

Interdisziplinäre neurovaskuläre Netzwerke: eine Standortbestimmung

Zusammenfassung

Hintergrund. Um das gesamte neurovaskuläre Erkrankungsspektrum auf einem hohen Qualitätsniveau abzubilden, das über die reine akute Schlaganfallversorgung hinausgeht, hat die Deutsche Schlaganfall-Gesellschaft (DSG) gemeinsam mit den Fachgesellschaften für Neurochirurgie und Neuroradiologie ein Zertifizierungsverfahren für sog. neurovaskuläre Netzwerke (NVN) entwickelt. Strukturell setzt sich ein NVN aus einem koordinierenden Zentrum mit mindestens 3 neurovaskulären Netzwerkpartnern mit einer zertifizierten Stroke-Unit zusammen. Nach diesem neuen Standard wurden von 2018 bis 2020 bisher 15 NVN auditiert und zertifiziert.
Fragestellung. Wie leistungsfähig sind die NVN? Werden hohe Standards eingehalten? Material und Methoden. Ausgewertet wurden die Auditberichte. Die Datenlage geht auf die Jahre 2017 bis 2019 zurück.

Ergebnisse. Die 15 NVN behandelten insgesamt 86.510 Schlaganfallpatienten im Erhebungsjahr und waren mit insgesamt 107 Partnerkliniken vernetzt, die durchschnittlich $25 \mathrm{~km}$ entfernt vom koordinierenden Zentrum lagen und insgesamt 2726 Patienten verlegten. In den koordinierenden Zentren wurden 2383 Patienten mit nichttraumatischen intrazerebralen Blutungen behandelt und 2463 Thrombektomien durchgeführt. Bei 712 Patienten mit akuten aneurysmatischen
Subarachnoidalblutungen wurde eine endovaskuläre Therapie und bei 401 ein Clipping vorgenommen. Die Auditierung verlief in der Mehrzahl der NVN erfolgreich. Diskussion. Der Zertifizierungsprozess der NVN ist erfolgreich gestartet und die Audits erwiesen sich als gutes Instrument der Qualitätskontrolle und -verbesserung. Die $15 \mathrm{NVN}$ sind leistungsfähig und behandeln mehr als ein Viertel der Schlaganfallpatienten in deutschen Stroke-Units.

Schlüsselwörter

Stroke-Unit · Thrombektomie · Clipping . Endovaskuläre Therapie · Interhospitale Kooperation

\section{Interdisciplinary neurovascular networks: state of the art}

\begin{abstract}
Background. In order to treat the complete spectrum of neurovascular diseases at a high level of quality, which goes beyond the purely acute treatment of stroke, the German Stroke Society (DSG) together with the German Societies for Neurosurgery and Neuroradiology developed a certification procedure for neurovascular networks (NVN). Structurally, a NVN consists of a coordinating center with at least three neurovascular network partners with a certified stroke unit From 2018 to 2020 a total of 15 NVN have so far been audited and certified according to this new standard.

Objective. How efficient are the NVN? Are high standards maintained?
\end{abstract}

Material and methods. The reports of the audits were analyzed. The data were taken from the period 2017-2019.

Results. The 15 NVN treated a total of 86,510 stroke patients in the years examined and were networked with a total of 107 partner clinics, which were situated an average of $25 \mathrm{~km}$ from the coordinating center and transferred a total of 2726 patients. The coordinating centers performed 2463 thrombectomies and treated 2383 patients with nontraumatic intracerebral bleeding. In 712 patients with acute aneurysmatic subarachnoid hemorrhages endovascular treatment was carried out and clipping in 401.
The audit was successful in the majority of the NVN.

Conclusion. The certification process of NVN has been successfully established and the audits proved to be a useful instrument for quality control and improvement. The $15 \mathrm{NVN}$ are highly efficient and treat more than one quarter of stroke patients in German stroke units.

Keywords

Stroke unit - Thrombectomy · Clipping . Endovascular treatment · Interhospital cooperation
2463 Thrombektomien (164 pro NVN; Spannweite 79-387). Die „door-to-needle time" betrug $32 \mathrm{~min}$ (23-42) und die „door-to-groin time“ $65 \mathrm{~min}$ (47-85).

Es wurden 2383 Patienten mit nichttraumatischen intrazerebralen Blutungen behandelt, von denen 635 operiert wurden. 712 Patienten mit akuten aneurysmatischen Subarachnoidalblutungen wurden endovaskulär und 401 mittels Clipping behandelt (Mittelwert 126/NVN; 94-177). Eine elektive endovaskuläre Aneurysmabehandlung erfolgte bei 738 und ein Clipping bei 255
Patienten. Ein Stenting einer Karotisstenose wurde bei 1128 Patienten und eine Karotisendarterektomie bei 1251 Patienten vorgenommen.

Darüber hinaus führten die neurochirurgischen Kliniken durchschnittlich 908 Kopf- und 700 Wirbelsäulenoperationen durch.

\section{Charakterisierung der Partner- kliniken der neurovaskulären Netzwerke}

Die 107 Partnerkliniken behandelten 64.158 ischämische Schlaganfallpatienten (durchschnittlich 625 pro Klinik; 28-1737). 69 der 107 Partnerkliniken verfügten über eine regionale StrokeUnit, 6 über eine überregionale und 32 über eine nichtzertifizierte Stroke-Unit oder eine internistische Abteilung mit Schlaganfallversorgung. 
Tab. 1 Charakteristik der koordinierenden Zentren ${ }^{a}$

\begin{tabular}{|c|c|}
\hline Anzahl Schlaganfälle der NVN & 86.510 \\
\hline Gesamtanzahl Schlaganfälle der koord. Zentren & 18.206 \\
\hline Anzahl Schlaganfälle/koord. Zentrum & $1214(895-1635)$ \\
\hline SU-Betten/koord. Zentrum & $13(8-18)$ \\
\hline Eigenständige Neuro-ITS & $8 / 15$ \\
\hline Anzahl der Thrombolysen/koord. Zentrum & $170(40-384)$ \\
\hline „Door-to-needle“-Zeit (min) & $32(23-42)$ \\
\hline Thrombektomien gesamt & 2463 \\
\hline Anzahl Thrombektomien/koord. Zentrum & $164(79-387)$ \\
\hline „Door-to-groin"-Zeit (min) & $65(47-85)$ \\
\hline Anzahl ICB/koord. Zentrum & $159(36-384)$ \\
\hline Anzahl Operierter ICB/koord. Zentrum & $52(12-123)$ \\
\hline Aneurysmatische $S A B /$ koord. Zentrum & $126(94-177)$ \\
\hline Karotis-Stenting/koord. Zentrum & $75(9-456)$ \\
\hline Karotisendarterektomie/koord. Zentrum & $83(34-184)$ \\
\hline \multicolumn{2}{|c|}{$\begin{array}{l}\text { ICB intrazerebrale Blutung, koord. Zentrum koordinierendes Zentrum, NVN neurovaskuläres } \\
\text { Netzwerk, SAB Subarachnoidalblutung, SU Stroke-Unit, ITS Intensivstation } \\
\text { a Zahlenangaben mit Klammern sind Mittelwert und Spannweite }\end{array}$} \\
\hline
\end{tabular}

\section{Tab. 2 Charakteristik der Partnerkliniken ${ }^{a}$}

\begin{tabular}{|l|l|}
\hline Anzahl der Partnerkliniken insgesamt & 107 \\
\hline Anzahl der Partnerkliniken/NVN & $7(3-16)$ \\
\hline Entfernung zum koordinierenden Zentrum (km) & $25(4-147)$ \\
\hline Anzahl Schlaganfälle der Partnerkliniken & 64.158 \\
\hline Anzahl Schlaganfälle/Partnerkliniken & $625(28-1737)$ \\
\hline Regionale Stroke-Units/Gesamtzahl der Partnerkliniken & $69 / 107$ \\
\hline Überregionale Stroke-Units/Gesamtzahl der Partnerkliniken & $6 / 107$ \\
\hline Nichtzertifizierte Kliniken/Gesamtzahl der Partnerkliniken & $32 / 107$ \\
\hline Anzahl verlegte Patienten ins koordinierende Zentrum & 2726 \\
\hline Anzahl verlegte Patienten/NVN & $182(64-541)$ \\
\hline Thrombektomien verlegt & 1015 \\
\hline ICB verlegt & 579 \\
\hline Aneurysmatische SAB verlegt & 373 \\
\hline Karotis-Stenting/Karotisendarterektomie verlegt & 268 \\
\hline Sonstige Verlegungen & 420 \\
\hline ICB intrazerebrale Blutung, NVN neurovaskuläres Netzwerk, SAB Subarachnoidalblutung \\
\hline aZahlenangaben mit Klammern sind Mittelwert und Spannweite
\end{tabular}

Durchschnittlich wurden im Erhebungsjahr 182 Patienten (64-541) ins koordinierende Zentrum des NVN verlegt: 1015 Patienten zur Thrombektomie, 579 Patienten mit einer intrazerebralen Blutung, 373 mit einer aneurysmatischen Subarachnoidalblutung, 263 zum Karotis-Stenting oder zur Endarterektomie und 420 aus anderweitigen Gründen (•Tab. 2). bezogen sich hauptsächlich auf folgende Themenbereiche:

- Optimierung/Erfassung der Prozesszeiten (DNT; „door-to-needle time“, Sekundärtransportzeiten),

- grenzwertige Anzahl von Partnerkliniken, zu geringe Anzahl von Zuweisungen,

- kein überzeugendes Konzept der Versorgung auf der Intensivstation (kein ausreichendes Weiterbildungskonzept auf der Intensivstation; unzulängliche SOP [„standard operating procedure"]),

- Intensivierung der Kooperation im Netzwerk (Fortbildungen, Netzwerktreffen),

- strukturelle Unstimmigkeiten (umliegende Kliniken nicht ausreichend integriert).

Abweichungen wurden ausgesprochen, wenn $\mathrm{KO}$ (Knock-out)-Kriterien oder andere Anforderungen, die zur Zertifizierung erfüllt werden müssen, nicht gewährleistet wurden. Die Abweichungen bezogen sich auf formale Versäumnisse (fehlende Kooperationsverträge, fehlendes Konzept der Versorgung intensivmedizinischer Patienten) oder eine zu geringe Anzahl an Partnerkliniken mit zertifizierter Stroke-Unit und konnten in einer Frist behoben werden.

\section{Diskussion}

In einem Zeitabschnitt von 2 Jahren wurden 15 neurovaskuläre Netzwerke auditiert und zertifiziert, die in dem Jahr der Datenerfassung zusammen mit ihren 107 Partnerkliniken über 86.000 Schlaganfallpatienten versorgten. 75 der 107 Partnerkliniken sind zertifizierte StrokeUnits, sodass zusammen mit den 15 koordinierenden Zentren fast $30 \%$ der StrokeUnits in Deutschland bereits einem NVN angeschlossen sind. Die NVN führten über 2400 Thrombektomien durch und behandelten über 700 Patienten mit akuten aneurysmatischen Subarachnoidalblutungen. Dies ist eine Leistungsbilanz, die ohne die seit über zwei Jahrzehnten fortentwickelte Versorgungsstruktur mit flächendeckend über 300 zertifizierten Stroke-Units und die parallel stetig weiterentwickelte neurointerventionelle 
und neurochirurgische Expertise nicht möglich wäre [5].

Die regionalen und überregionalen Stroke-Units sind der Grundstock der Schlaganfallversorgung - nicht nur der ischämischen Schlaganfälle, sondern auch der intrazerebralen Blutungen, Gefäßmissbildungen und Subarachnoidalblutungen, die interdisziplinär gemeinsam mit den neurochirurgischen und neuroradiologischen Abteilungen versorgt werden. Parallel dazu wurden seitens der Neuroradiologie die personellen und infrastrukturellen Voraussetzungen für eine 24/7(24 Stunden/7 Tage)-Bereitschaft zur interventionellen Behandlung des akuten Schlaganfalls (mechanische Thrombektomie) und zerebraler Gefäßmalformationen flächendeckend etabliert. Dies verkürzt die Transportzeiten, wenn der Dienst besetzt ist, erfordert aber weiterhin die Kooperation mit einem Zentrum der 24/7-Versorgung. Für Patienten mit akuten aneurysmatischen Subarachnoidalblutungen, raumfordernden Hirninfarkten oder intrazerebralen Blutungen ist ohnehin eine enge Zusammenarbeit notwendig.

\section{》) Die NVN gewährleisten eine flächendeckende Versorgung auf hohem Niveau}

Die Etablierung einer dritten Versorgungsebene mit einer hochspezialisierten Versorgung in einem koordinierenden Zentrum mit Netzwerkstruktur profitiert von verlässlichen Kooperationsabsprachen, gemeinsamen SOPs, regelmäßigen Netzwerktreffen zur Problemlösung und der Erfassung von Qualitätsparametern.

Die NVN mit ihren kooperierenden Partnerkliniken unterscheiden sich hinsichtlich ihrer Struktur. Die koordinierenden Zentren sind ausnahmslos leistungsfähige Maximalversorger und Universitätskliniken, die die gesamte $\mathrm{Pa}$ lette der spezialisierten neurovaskulären Versorgung anbieten. Sie kooperieren in Netzwerken, die von kleinen NVN mit 3 Partnerkliniken mit regionalen StrokeUnits bis zu sehr großen NVN mit einer Vielzahl von zuweisenden, zertifizierten und nichtzertifizierten Kliniken im ländlichen Raum reicht. Die Intensität der Netzwerkkooperation zeigt sich in der Anzahl der Zuweisungen, die von 64 bis 541 Patienten pro Jahr reicht (Mittelwert 182). Besonders Netzwerke in Ballungsgebieten mit vielen alternativen Kooperationspartnern weisen teils eine geringe Anzahl gemeinsamer $\mathrm{Pa}$ tienten auf, was sich aufgrund schlecht eingespielter Abläufe nachteilig auf die Versorgungsqualität auswirken kann. Eine Kooperation mit verschiedenen Zentren führt zu einer Vervielfachung von Schnittstellenproblemen, sodass eine gewisse "Netzwerktreue“ in einem NVN zu fordern ist.

Die Partnerkliniken der koordinierenden Zentren in den NVN sind teilweise sehr heterogen. Das Spektrum reicht dabei von großen Kliniken mit einer (über-)regionalen Stroke-Unit und weit über 1000 Schlaganfallpatienten und einer neuroradiologischen und/ oder neurochirurgischen Abteilung, die das Netzwerk als Back-up für Engpässe nutzen, bis hin $\mathrm{zu}$ internistischen Kliniken mit weniger als 100 Schlaganfallpatienten im Jahr, die teilweise in Tele-Netzwerke eingebunden sind.

\section{) Im Audit werden individuelle Versorgungsstrukturen kritisch hinterfragt}

Das Audit bietet dabei einen idealen Rahmen, um diese individuellen Versorgungsstrukturen im direkten Gespräch kritisch zu hinterfragen und auf den Einzelfall zugeschnittene Vorschläge $\mathrm{zu}$ unterbreiten, u.a.:

- Ist es sinnvoll, Schlaganfallpatienten in einer internistischen Klinik telemedizinisch $\mathrm{zu}$ versorgen, wenn eine zertifizierte Stroke-Unit in $15 \mathrm{~km}$ Entfernung erreichbar ist?

- Sollte eine internistische Klinik nicht besser in das Netzwerk integriert werden, um einen Einblick in die dortige Versorgung zu erhalten und eine Kooperation zu etablieren?

- Wie kann das koordinierende Zentrum mit seinen Netzwerkpartnern dafür Sorge tragen, dass Schlaganfälle nicht mehr in internistische
Abteilungen, sondern in die nächste Stroke-Unit verbracht werden?

In nahezu allen Netzwerken war die teleradiologische Anbindung gut gelöst und oftmals wurden, unterstützt durch die Zertifizierung, noch bestehende ITProbleme beseitigt.

Wie immer, wenn eine Struktur von externen Fachauditoren evaluiert wird, werden neben Stärken auch Schwächen erkannt. Eine häufige Bemerkung betraf die Versorgung neurologischer und neurochirurgischer Patienten auf Intensivstationen. Vor dem Hintergrund der strengen Strukturvorgabe für Intensivstationen (OPS 8-98f - aufwendige intensivmedizinische Komplexbehandlung), die auch von einigen Universitätskliniken nicht mehr vollumfänglich gewährleistet werden kann, erfolgt die Betreuung zunehmend auf interdisziplinären und anästhesiologisch geführten Intensivstationen. Dies muss nicht zwangsläufig zum Nachteil der neurologischen/neurochirurgischen Patientenversorgung sein, sofern die ärztliche Betreuung seitens neurologischer und neurochirurgischer Ober-/Fachärzte mit der intensivmedizinischen Weiterbildung nach einem strukturierten Konzept engmaschig erfolgt und ausreichend Assistenz- und Fachärzte auf den Intensivstationen weitergebildet werden.

》) Voraussetzung für das Zertifikat ist eine intensive Kooperation der Netzwerkpartner

Die Entscheidungsprozesse bei der Versorgung schwer erkrankter neurovaskulärer Patienten sind komplex und bedürfen standardisierter Prozeduren, um optimale Ergebnisse zu erzielen. Dies beginnt bei der rechtzeitigen Identifizierung eines Patienten, der einer Verlegung in das koordinierende Zentrum bedarf (Bildgebung; u.a. Computertomographie[CT]-Angiographie), der Organisation des Sekundärtransports und der verlässlichen Übernahme des Patienten. Diese Versorgungskette ist komplex („Door-to-door“-/,Time-toleave“-Zeiten in der verlegenden Klinik), anfällig für Störfaktoren (Transportfra- 
ge - luft-/bodengebunden) und bedarf fortwährender Optimierung [6]. Voraussetzung für das Zertifikat ist daher ein regelmäßiger Kontakt der Netzwerkpartner mit Fallbesprechungen, Diskussion der Prozesszeiten und Morbiditäts-/ Mortalitätskonferenzen, um Transparenz zu schaffen und die Netzwerkprozesse zu optimieren.

Die Netzwerkstruktur kann auch dazu genutzt werden, in der Diskussion mit den Netzwerkpartnern die endovaskuläre Versorgung in kleinen Kliniken mit geringen Thrombektomiezahlen selbstkritisch zu hinterfragen, in einem gelebten Netzwerk gemeinsam an Innovationen teilzunehmen und die Behandlungsergebnisse einer netzwerkinternen Prüfung zu unterziehen.

\section{》) Eine adäquate Finanzierung der spezialisierten Leistungen ist erforderlich}

Kritisch zu betrachten ist, dass eine ganze Reihe von Leistungen und Maßnahmen innerhalb des NVN bisher nicht über das DRG(„diagnosis related groups“)System abgebildet wird. Für eine langfristig tragfähige Weiterentwicklung der NVN ist eine adäquate Finanzierung der spezialisierten Leistungen jedoch essenziell. Die Autoren setzen daher große Hoffnung in die Ausweitung der vom Gemeinsamen Bundesausschuss (GBA) beschlossenen Zentrumszuschläge auf die neurovaskuläre Medizin. Zertifizierte NVN qualifizieren sich in prototypischer Weise für diese Struktur und deren Rationale. Erste Abstimmungsgespräche sind positiv verlaufen.

Die vorliegende Datenanalyse hat Schwächen und Limitationen, die sich aus der Erhebung nicht nachprüfbarer Krankenhausdaten ergeben. Die Zahlenangaben spiegeln die Netzwerkrealität wider, erkennend, dass Daten zum Verhältnis von Karotis-Stenting zu Endarterektomie oder Clipping und Coiling von Aneurysmen nicht zwangsläufig den Zahlen aus großen Registern entsprechen. Andere Kennzahlen, die wichtige Qualitätsparameter in einem NVN sind, wie Sekundärtransportzeiten oder die Anzahl von Übernahmeablehnungen, wurden bisher nicht verlässlich erhoben und sollen künftig mehr im Fokus stehen.

\section{Fazit für die Praxis}

- Zertifizierte neurovaskuläre Netzwerke (NVN) stellen eine neue, qualitätsgesicherte Struktur dar, um das gesamte Spektrum der neurovaskulären Medizin auf hohem fachlichem Niveau abzudecken. Durch die gelebte Kooperation mit intensivierter, interdisziplinärer Abstimmung zwischen den NeuroFächern wird die Fachexpertise gebündelt und die Qualität gesteigert. NVN schaffen mehr Transparenz und Verbindlichkeit in der interhospitalen Kooperation und verleihen der vaskulären Neuromedizin insgesamt mehr Gewicht.

- Die zertifizierte Netzwerkstruktur trägt dazu bei, Schnittstellenprobleme zu erkennen und zu beheben. Ein breites Spektrum von Klinikpartnern profitiert von einem NVN: von der überregionalen Stroke-Unit, die das NVN für das Überbrücken von Versorgungsengpässen in der endovaskulären Versorgung benötigt, bis hin zur nichtzertifizierten internistischen Klinik im ländlichen Umfeld, die einen leistungsstarken Kooperationspartner an ihrer Seite weiß.

\section{Korrespondenzadresse}

\section{Prof. Dr. J. Röther}

Kopf- und Neurozentrum, Neurologische Abteilung mit überregionaler Stroke Unit, Neurophysiologie und Neurologischer Intensivmedizin, Asklepios Klinik Hamburg Altona, Asklepios Campus Hamburg der Semmelweis Universität

Paul-Ehrlich-Straße 1, 22763 Hamburg,

Deutschland

j.roether@asklepios.com

\section{Einhaltung ethischer Richtlinien}

Interessenkonflikt. J. Röther, O. Busse, A. Berlis, A. Dörfler, C. Groden, G. Hamann, O. Jansen, J. Meixensberger, O. Müller, J. Regelsberger, H. Steinmetz, H. Vatter, W. Weber und D. Nabavi geben an, dass kein
Interessenkonflikt im Zusammenhang mit den NVN besteht.

Für diesen Beitrag wurden von den Autoren keine Studien an Menschen oder Tieren durchgeführt. Für die aufgeführten Studien gelten die jeweils dort angegebenen ethischen Richtlinien.

\section{Literatur}

1. Busse O, Rother J, Faiss J, Hamann GF, Hupp T, Jansen $O$ et al (2013) interdisciplinary neurovascular network: a new structure for treatment of stroke and other cerebrovascular diseases in germany. Nervenarzt 84:1228-1232

2. Goyal M, Menon BK, van Zwam WH, Dippel DW, Mitchell PJ, DemchukAM et al (2016) Endovascular thrombectomy after large-vessel ischaemic stroke: a meta-analysis of individual patient data from five randomised trials. Lancet 387:1723-1731

3. Ringleb P, Hamann G, Röther J, Jansen O, Groden C, Veltkamp R (2016) S2 Leitlinie Akuttherapie des ischämischen Schlaganfalls - Ergänzung 2015 - Rekanalisierende Therapie. Akt Neurol 43(02):82-91

4. Eyding J et al (2012) Das „Neurovaskuläre Netz Ruhr" - Konzeption und praktische Umsetzung eines dezentralen Netzwerkes zur Optimierung einer flächendeckenden neurovakulären Maximalversorgung akuter Schlaganfallpatienten in der Metropolenregion Ruhrgebiet. Akt Neurol 39:1-8

5. Nabavi DG, Koennecke $H$, Ossenbrink $M$ et al (2019) Zertifizierungskriterien für Stroke-Units in Deutschland. Nervenarzt 90:335-342. https://doi. org/10.1007/s00115-018-0633-y

6. Holodinsky JK, Kamal N, Wilson AT, Hill MD, Goyal M (2017) Workflow in acute stroke: What is the 90th percentile? Stroke 48:808-812 